\title{
VARIACIÓN DIARIA DE CARBONO NEGRO Y OZONO TOTAL EN ZONA
} DE UNTELS.

\author{
${ }^{1}$ Odón R. Sánchez Ccoyllo Mdoi , , ${ }^{1}$ Yeiko Gala Barraza, ${ }^{1}$ Angela Huacha \\ Paucarpura, ${ }^{1}$ Jessica Arratea Morán \\ ${ }^{1}$ Universidad Nacional Tecnológica de Lima Sur, Lima, Perú
}

Recibido: 05/09/2021 Revisado: 03/10/2021 Aceptado: 16/11/2021 Publicado: 30/01/2022

\section{Resumen}

El objetivo de la presente investigación es estudiar variación temporal diaria de Carbono Negro (BC) y ozono diario de la columna total en área definida en un cuadrado $(77.2 \mathrm{~W}$, 12.48S, 76.6W, 11.95S) centrado del cuadrado en UNTELS en el periodo de 21 de abril de 2020 a 10 de junio de 2020. Adicionalmente, la finalidad de esta pesquisa es realizar análisis de correlación de Pearson entre temperatura del aire, BC y ozono de la columna total de la atmosfera en la misma zona de UNTELS y periodo de estudio. Los valores horarios de $\mathrm{BC}$ y ozono diario total se han obtenido de los datos científicos diarios de la página de la NASA disponible de manera gratuita en el portal de la NASA-Giovanni. Los valores horarios de $\mathrm{BC}$ se han obtenido de datos de re-análisis de MERRA-2 (sigla de inglés, Modern-Era Retrospective analysis for Research and Applications, Version 2). Los valores diarios de ozono atmosférico de la columna total se han obtenido medidos con el sensor AIRS (sigla de inglés Atmospheric Infrared Sounder) a bordo de satéliteAqua. Mientras, temperatura del aire ha sido medido usando el equipo meteorológico portátil en UNTELS. Los resultados indican que los valores diarios de BC y ozono total en la zona de UNTELS varia de 194 a $368 \mathrm{ng} / \mathrm{m}^{3}$, y de 230 a 251 unidad de Dobson respectivamente.

\section{Palabras claves}

AIRS, UNTELS, SATELITE-AQUA, MERRA-2 


\begin{abstract}
The objective of this research is to study daily temporal variation of Black Carbon (BC) and daily ozone of the total column in defined area in a square $(77.2 \mathrm{~W}, 12.48 \mathrm{~S}, 76.6 \mathrm{~W}$, 11.95S) centered on the square in the UNTELS in the period from April 21, 2020 to June 10, 2020. Additionally, the purpose of this research is to perform a Pearson correlation analysis between air temperature, $\mathrm{BC}$ and ozone of the total column of the atmosphere in the same area of UNTELS and study period. The hourly values of BC and total daily ozone have been obtained from the daily scientific data of the NASA page available for free on the NASA-Giovanni portal. Hourly BC values were obtained from MERRA-2 (Modern-Era Retrospective analysis for Research and Applications, Version 2) reanalysis data. The daily values of atmospheric ozone of the total column have been obtained measured with the AIRS sensor (Atmospheric Infrared Sounder) on board the Satellite-Aqua. Meanwhile, air temperature has been measured using portable meteorological equipment at UNTELS. The results indicate that the daily values of BC and total ozone in the UNTELS zone vary from 194 to $368 \mathrm{ng} / \mathrm{m}^{3}$, and from 230 to 251 Dobson units respectively.
\end{abstract}

\title{
Key Words
}

AIRS, UNTELS, SATELITE-AQUA, MERRA-2 


\section{Introducción}

Los aerosoles atmosféricos pueden modificar el balance de radiación a través de los efectos directos e indirectos de los aerosoles (Kumar, 2014). Un componente importante de material particulado fino $\left(\mathrm{PM}_{2.5}\right)$ es Carbono Negro ( $\mathrm{BC}$ sigla de inglés). $\mathrm{BC}$ es principalmente generado por la combustión incompleto de combustible diésel en áreas urbanas (Zhao et al., 2021). BC causa cáncer (Sitnov et al., 2020). Por tanto, es necesario descripción de la variación temporal de BC en la zona de UNTELS. Por esto, se ha utilizado dato de re-análisis de MERRA-2 (sigla de ingés, Modern-Era Retrospective analysis for Research and Applications, Version 2) lo cual es realizado por la NASA (Qin et al., 2019) para el periodo de 21 de abril a 10 de junio de 2020, debido que para ese periodo se ha hecho medición temperatura del aire con el equipo meteorológico "DAVIS vantage pro 2 plus" en UNTELS en el marco de proyecto de investigación en UNTELS llamado "Análisis Morfológico, Metales y Origen de las Partículas Respirables en la Zona Sur de Lima”.

Otro contaminante que perjudica la salud de la población es la concentración de ozono a nivel de suelo ya que ozono a nivel de superficie es un contaminante tóxico que puede causar daño al pulmón de las personas y también daño a las plantas (Rajab et al., 2013). Mientras, el ozono en la estratosfera es bueno para los seres vivos y otros seres vivos en la tierra ya que el ozono en ese nivel absorbe la radiación ultravioleta-B (Rajab et al., 2013). En ese sentido en este estudio se ha utilizado el dato de ozono de la columna total de la atmósfera medido por el instrumento AIRS (sigla de inglés Atmospheric Infrared Sounder) realizado por la NASA y de libre acceso en la página portal de Giovanni (https://giovanni.sci.gsfc.nasa.gov/giovanni/). El instrumento AIRS está a bordo de satélite Aqua de la NASA (Rajab et al., 2013).

El objetivo del presente estudio es analizar variación diaria de carbono negro (BC) y columna de ozono total en la zona de UNTELS para el periodo de 21 de abril a 10 de junio de 2020. También, el objetivo de este estudio es realizar análisis de correlación de Pearson entre temperatura del aire, BC y ozono de la columna total de la atmosfera en la zona de UNTELS. 


\section{Materiales y Métodos}

Ha sido obtenido los valores horarios de BC promedio para la zona de UNTELS a través de la página de la NASA de acceso libre y gratuito. Esto es, promedio para un cuadrado, centrado en UNTELS. Las coordenadas del área de cuadrado fueron las siguientes: 77.2W, 12.48S, 76.6W, 11.95S (Figura 1). Esos valores de BC horarias han sido obtenido de la página de la NASA, específicamente de la página de Giovanni que se mencionan a continuación: https://giovanni.sci.gsfc.nasa.gov/giovanni/. En esa página web se ha obtenido promedio de área de los valores horarios de la concentración en masa a nivel de superficie de BC en el periodo de 21 de abril de 2020 a 10 de junio de 2020. Luego se ha calculado valores diarios de $\mathrm{BC}$ utilizando el programa $\mathrm{R}$. Los valores horarios de $\mathrm{BC}$ se encuentran con resolución espacial de $0.5^{0}$ x $0.625^{0}$ resultado de modelo MERRA-2. Dicho proyecto MERRA-2 está disponible en web siguiente: https://gmao.gsfc.nasa.gov/reanalysis/MERRA-2/. Finalmente, se ha obtenido el promedio de área de la zona de UNTELS mencionado arriba (Figura1) de columna de ozono total diaria en el periodo de 21 de abril de 2020 a 10 de junio de 2020. Dicho valor de ozono se ha obtenido de instrumento AIRS, también disponible en la página de Giovanni https://giovanni.sci.gsfc.nasa.gov/giovanni/.

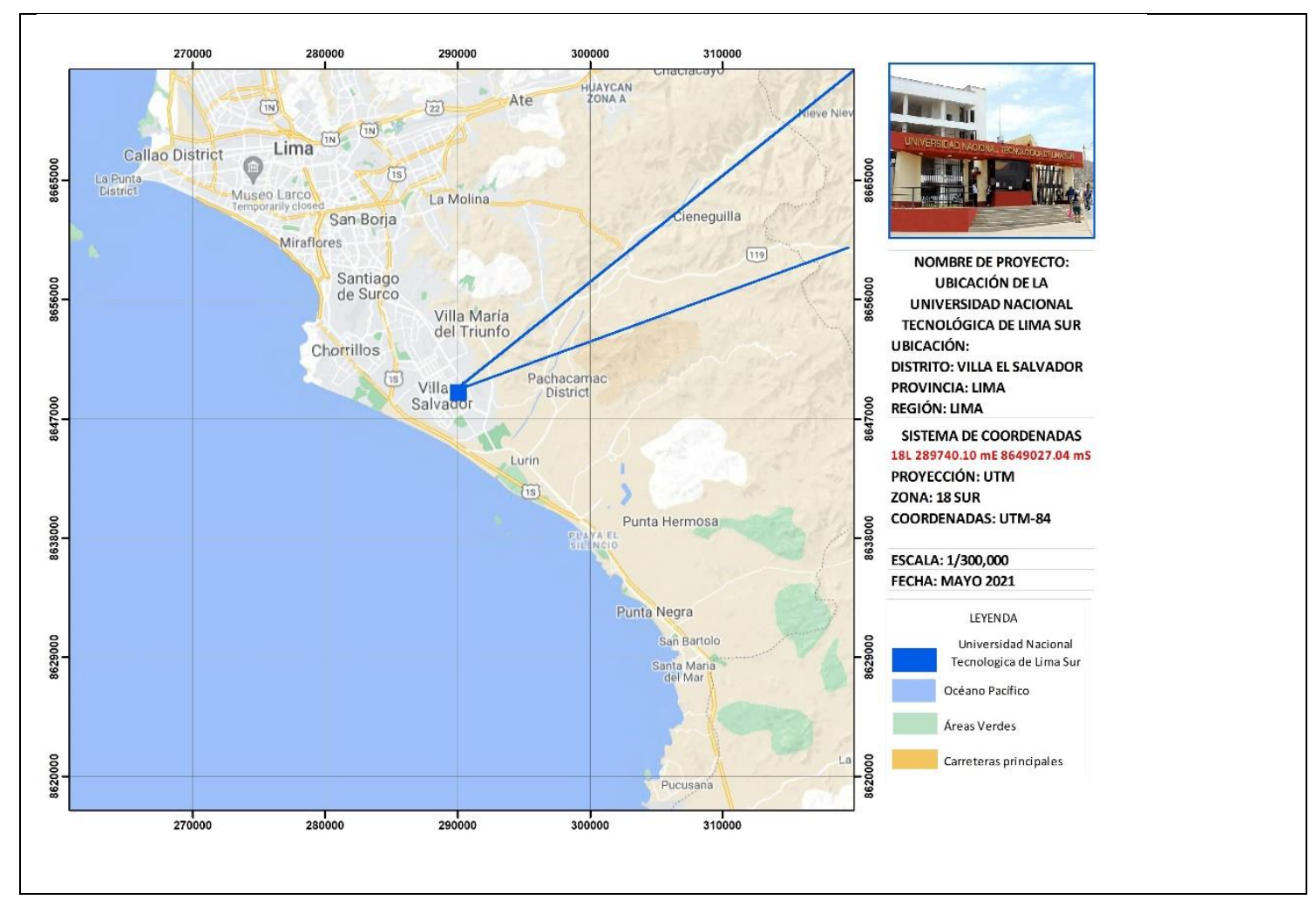


Figura 1. Ubicación de zona de estudio en UNTELS (cuadrado con color azul)

\section{Resultados y Discusiones}

Elemento químico que representa el aerosol fino es llamado carbono negro (BC). El BC a nivel de superficie en la zona de UNTELS varía de 194 a $368 \mathrm{ng} / \mathrm{m}^{3}$, con un promedio para el periodo de estudio de $255 \mathrm{ng} / \mathrm{m}^{3}$. BC se genera principalmente en los vehículos que usan diésel. Justo el valor más alto de BC ha alcanzado el día 30 de mayo de 2020 (Figura 2).

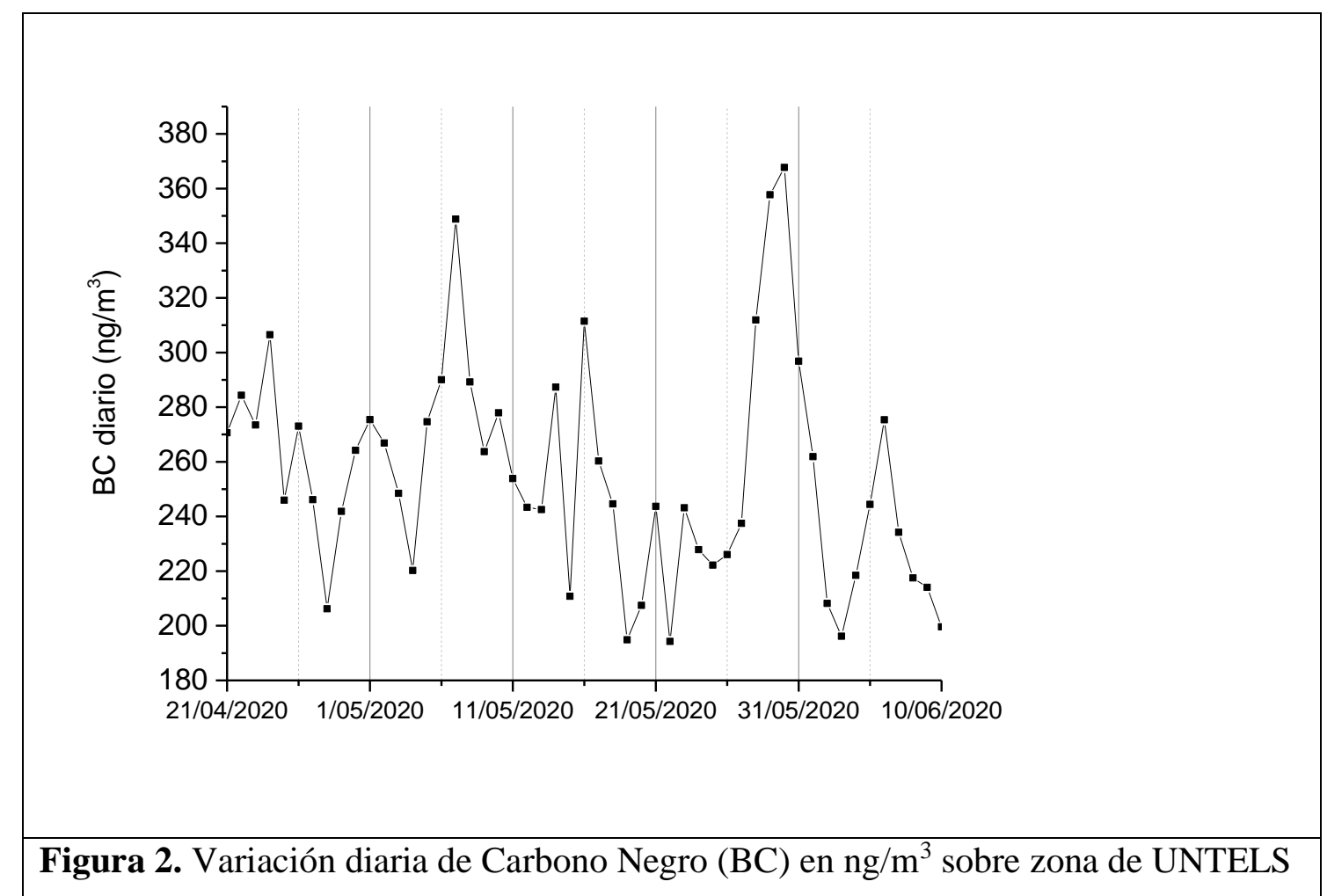

Para entender el valor elevado de BC el día 30 de mayo de 2020 se ha realizado el cálculo de trayectoria de llegada en UNTELS, justamente el día 30 de mayo en tres niveles de la atmosfera: 1000 m, 2000 m y 3000 m por arriba de suelo (figura 3). Para los tres niveles, las trayectorias llegan a UNTELS con dirección de NNW (Norte-Norte-Oeste). El origen de la trayectoria de $1000 \mathrm{~m}$ se origina con 24 días de antecedencia en Océano Pacifico al frente de norte de Lima, luego las parcelas de aire se dirigen a Lima, para luego llega a Lima en UNTELS (Figura 3). El origen de la trayectoria de $2000 \mathrm{~m}$ se nota que se origina 
en litoral de Océano Pacifico en norte de Lima, para luego dichas parcelas de aire se dirigen para Lima, llegando en UNTELS con trayectoria de NNW (Figura 3). El origen de la trayectoria de $3000 \mathrm{~m}$ se origina en Océano Pacifico en frente de Lima norte, luego la parcela de aire se dirige al sur, para luego llegar a Lima en UNTELS también con dirección de trayectoria NNW a 3000 m (Figura 3).

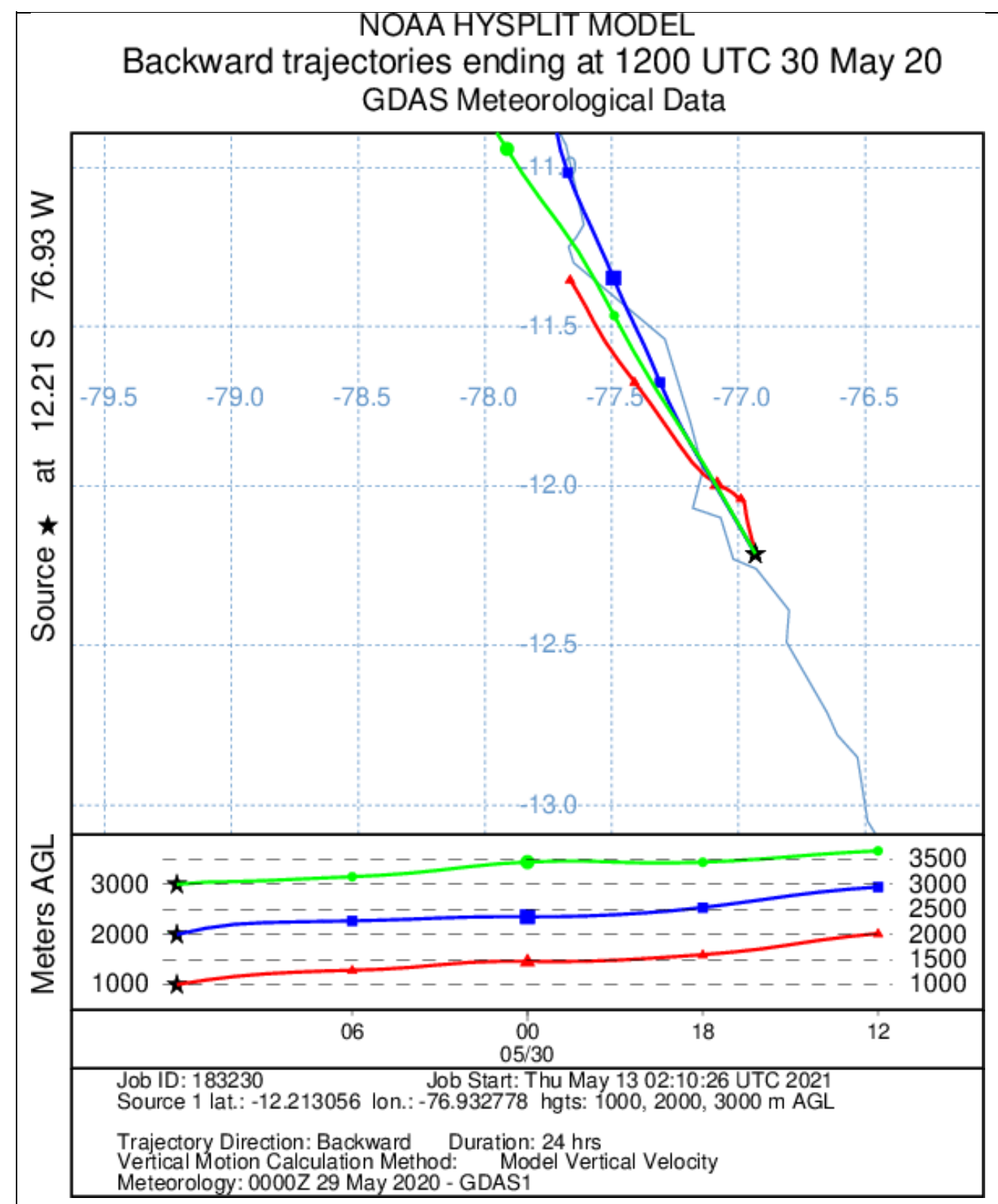

Figura 3. Trayectoria de llegada en UNTELS el día 30 de mayo de 2020. Color rojo indica que la trayectoria llega a $1000 \mathrm{msnm}$; color azul indica que la trayectoria llega a $2000 \mathrm{msnm}$; color verde indica que la trayectoria llega a $3000 \mathrm{msnm}$. 
También, para entender variación diaria de $\mathrm{BC}$ se ha realizado correlación de Pearson usando temperatura del aire medida en UNTELS de 21 de abril a 10 junio de 2020, ozono de la comuna total y BC para el mismo periodo temporal respectivamente (Tabla 1). Se nota una correlación positiva entre temperatura del aire y carbono negro de 0.371 para valor de $\mathrm{P}$ igual a 0.01 , esto indica que con mayor valor de temperatura el valor de $\mathrm{BC}$ pueda ser mayor (Figura 4). También se nota una correlación positiva de 0.581 entre ozono de la columna total y temperatura del aire en la zona de UNTELS con el valor de P igual a 0.05. Esto indica una correlación significativa entre el ozono de la columna total y temperatura del aire de manera positiva que puede ocurrir a mayor valor de la temperatura del aire un mayor valor de ozono de la columna total en la zona de UNTELS (Figura 5).

Tabla 1. Correlación de Pearson entre temperatura del aire, carbono negro y ozono de la columna total

\begin{tabular}{|l|l|l|l|}
\hline & Temperatura $\left({ }^{0} \mathrm{C}\right)$ & $\begin{array}{l}\text { Carbono Negro } \\
\left(\mathrm{ng} / \mathrm{m}^{3}\right)\end{array}$ & $\begin{array}{l}\mathrm{O}_{3} \text { columna total } \\
(\mathrm{DU})\end{array}$ \\
\hline Temperatura $\left({ }^{0} \mathrm{C}\right)$ & 1 & $0.371^{* *}$ & $0.581^{*}$ \\
\hline $\begin{array}{l}\text { Carbono Negro } \\
\left(\mathrm{ng} / \mathrm{m}^{3}\right)\end{array}$ & $0.371^{* *}$ & 1 & 0.082 \\
\hline $\begin{array}{l}\mathrm{O}_{3} \text { columna total } \\
(\mathrm{DU})\end{array}$ & $0.581^{*}$ & 0.082 & 1 \\
\hline
\end{tabular}

* en el nivel de 0.05 es significativa la correlación.

** en el nivel de 0.01 es significativa la correlación.

DU (sigla de inglés $)=$ Unidad de Dobson 


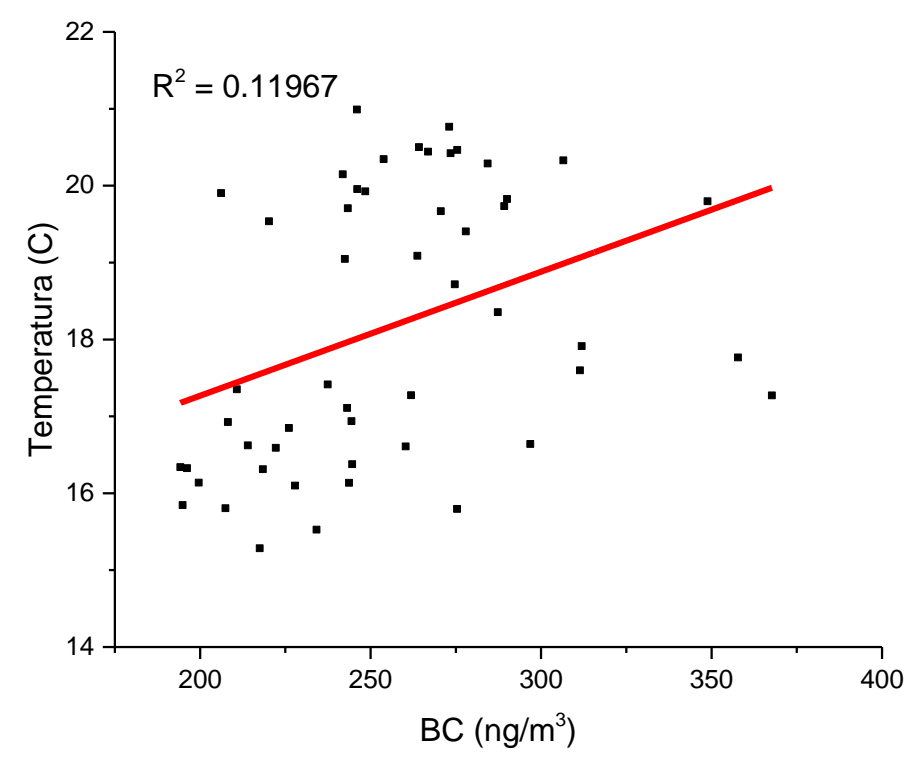

Figura 4. Dispersión de temperatura del aire y carbono negro (BC) en el periodo de 21 de abril de 2020 a 10 de junio de 2020

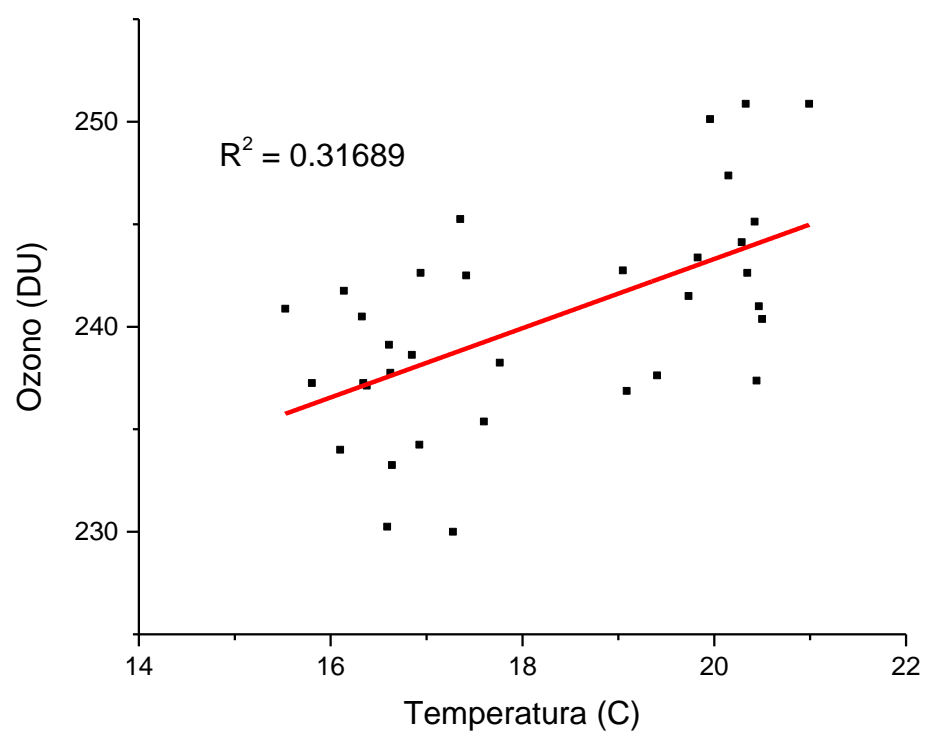

Figura 5. Dispersión entre columna de ozono total y temperatura del aire 


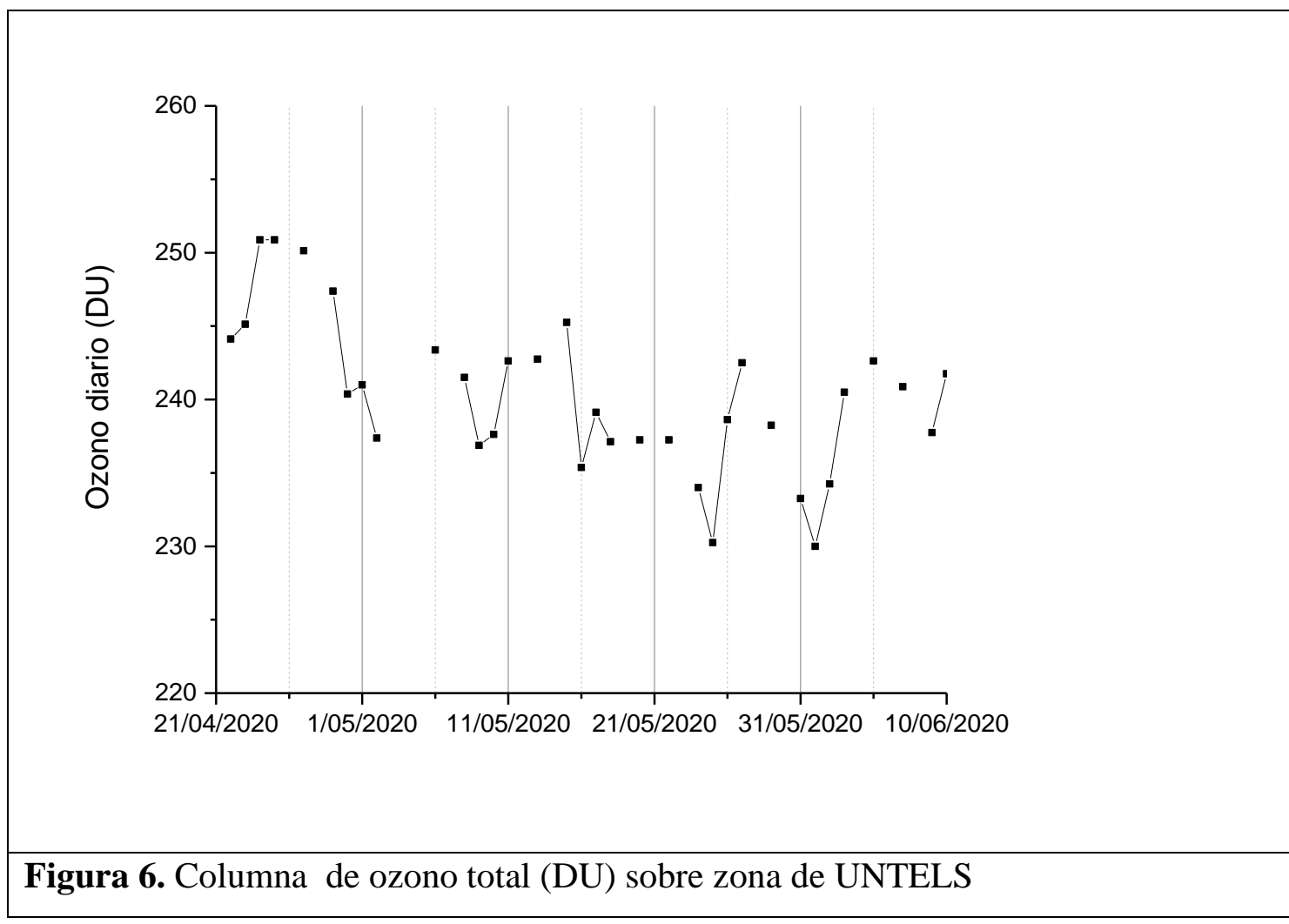

El valor de ozono de la columna total en la zona de UNTELS varia de 230 a $251 \mathrm{DU}$ (Unidad de Dobson). Unidad de Dobson significa valor de ozono en toda la columna de la atmosfera. El valor promedio de ozono diario en área de UNTELS es 240 DU. Este valor típico en el Perú en términos de media ozono total de la atmosfera.

\section{Conclusiones}

Por primera vez se ha realizado el análisis de variación diaria de carbono negro y ozono total diaria de la columna de la atmosfera utilizando datos científicos de la NASA en UNTELS en el periodo de 21 de abril a 10 de junio de 2020 ya que para ese periodo hubo medición de la temperatura del aire en UNTELS. Los resultados mostraron que para el día más alto de carbono negro diario para el periodo de 21 de abril a 6 de junio de 2020 en la zona de UNTELS que las trayectorias de llegada de parcelas de aire en tres niveles 
arriba de suelo en UNTELS (1000 m, $2000 \mathrm{~m}$ y $3000 \mathrm{~m}$ ) llegaron a UNTELS con trayectorias de dirección Norte-Norte-Oeste (NNW).

Por otro lado, el carbono negro (BC) que se genera de vehículos que utilizan diésel. En ese sentido utilizando datos científicos horarios de BC de la NASA, en la zona de UNTELS, BC tuvo un promedio de $255 \mathrm{ng} / \mathrm{m}^{3}$ lo cual es un valor aparentemente menor que los valores típicos en áreas urbanas. Por tanto, se recomienda para futuros estudios realizar el estudio de la variación temporal de BC observacional, teniendo en cuenta que los equipos que miden el BC es muy caro para comprar e infelizmente UNTELS no tiene equipo para medir BC.

Correlación de Pearson usando temperatura del aire medida en UNTELS de 21 de abril a 10 junio de 2020, ozono diario de la comuna total y BC diarios para el mismo periodo. Se encontró una correlación positiva de 0.371 para valor de $\mathrm{P}$ igual a 0.01 entre temperatura del aire diaria y $\mathrm{BC}$ diario. También se mostró una correlación positiva de 0.581 entre ozono diario de la columna total y temperatura del aire diaria en la zona de UNTELS con el valor de P igual a 0.05 .

\section{Referencias Bibliográficas}

Kumar, A. (2014). Long term (2003-2012) spatio-temporal MODIS (Terra/Aqua level 3) derived climatic variations of aerosol optical depth and cloud properties over a semi arid urban tropical region of Northern India. Atmospheric Environment, 83, 291-300. https://doi.org/10.1016/j.atmosenv.2013.10.030

Qin, W., Zhang, Y., Chen, J., Yu, Q., Cheng, S., Li, W., Liu, X., \& Tian, H. (2019). Variation, sources and historical trend of black carbon in Beijing, China based on ground observation and MERRA-2 reanalysis data. Environmental Pollution, 245(2), 853-863. https://doi.org/10.1016/j.envpol.2018.11.063

Rajab, J. M., Lim, H. S., \& Matjafri, M. Z. (2013). Monthly distribution of diurnal total column ozone based on the 2011 satellite data in Peninsular Malaysia. Egyptian Journal of Remote Sensing and Space Science, 16(1), 103-109. https://doi.org/10.1016/j.ejrs.2013.04.003

Sitnov, S. A., Mokhov, I. I., \& Likhosherstova, A. A. (2020). Exploring large-scale black-carbon air pollution over Northern Eurasia in summer 2016 using MERRA-2 
reanalysis data. Atmospheric Research, 235(November 2019), 104763.

https://doi.org/10.1016/j.atmosres.2019.104763

Zhao, J., Liu, Y., Shan, M., Liang, S., Cui, C., Chen, L., Gao, S., Mao, J., Zhang, H., Sun, Y., Ma, Z., \& Yu, S. (2021). Characteristics, potential regional sources and health risk of black carbon based on ground observation and MERRA-2 reanalysis data in a coastal city, China. Atmospheric Research, 256(February), 105563. https://doi.org/10.1016/j.atmosres.2021.105563 